\title{
The Perception and Satisfaction Survey of Human Anatomy Dissection Course Taught by Health Science Departments: Biomedical Laboratory Science, Physical Therapy, and Occupational Therapy
}

\author{
Se-Hoon Jung ${ }^{1}$, Seung-Ju Ahn ${ }^{2}$

\section{보건의료계열 학생들의 해부학 교육 개선을 위한 인식 및 만족도 조사: 임상병리학과, 물리치료학과, 작업치료학과 중심으로}

정세훈 ${ }^{1}$, 안승주 ${ }^{2}$

${ }^{1}$ 대구파티마병원 신경과, ${ }^{2}$ 대구보건대학교 임상병리과

\begin{abstract}
Human anatomy courses are one of the most fundamental subjects taught to students in any health-related science field. To improve the quality and to enlarge the scope of human anatomy courses, 130 students (Biomedical Laboratory Science, Physical Therapy, and Occupational Therapy) were given a questionnaire survey. The students were asked question regarding their anatomy course and cadaver dissection class. Specific questions regarding knowledge acquisition before and after the course was queried, in addition to their views on the necessity of cadaver dissection. The survey results indicated that students of all three departments were satisfied with the anatomy course, with no statistical difference in the students' sex or department affiliation. Although there was no difference between the three majors with respect to understanding the course materials, Biomedical Laboratory Science students showed higher satisfaction in this course $(p<0.05)$. Most students, regardless of sex and high school education, stated that cadaver dissection is an important part of the anatomy course. In conclusion, anatomy courses should implement cadaver dissection and actual practice rather than textbook-related studies alone.
\end{abstract}

Key words: Human anatomy, Health Science, Cadaver dissection

This is an Open Access article distributed under the terms of the Creative Commons Attribution Non-Commercial License (http://creativecommons.org/licenses/by-nc/4.0) which permits unrestricted non-commercial use, distribution, and reproduction in any medium, provided the original work is properly cited.

Copyright (C 2017 The Korean Society for Clinical Laboratory Science. All rights reserved.
Corresponding author: Se-Hoon Jung Department of Neurology, Daegu Fatima Hospital, 99 Ayang-ro, Dong-gu, Daegu 701-724, Korea

Tel: 82-53-940-7789, 7790

Fax: 82-53-954-7417

E-mail: jjhaha7@hanmail.net

Received: November 13, 2017

Revised $1^{\text {st: }}$ November 20, 2017

Revised $2^{\text {nd }}$ : November 21, 2017

Revised $3^{\text {rd: }}$ : November 22, 2017

Accepted: November 22, 2017
서 론

최근 건강 관련의 산업의 기술적 발달로 인해 보건의료인을 양성하는 대학의 교육은 아주 중요하다고 할 수 있다. 그중 인체 의 구조와 기능을 이해하는 해부학 수업은 보건 관련 학생들에
게 아주 중요하며 복잡한 인체의 해부학의 이해를 위한 실습의 중요성 및 체계적 교육은 필수라 할 수 있다[1].

보건의료를 전공하고 있는 학생들이 면허 시험 및 자격증 시 험을 위해 학과들의 전공과목과 기초과목인 해부학은 필수적이 며 교육 개선을 위한 보건의료계의 해부학 교육의 개선을 위해 
서 학년별 카데바실습의 만족도 및 이해도의 연구가 진행되었 고 통계적으로 학생들의 카데바 실습(cadaver dissection) 및 육안해부학(gross anatomy)의 교육시간의 요구도가 있었다 [2]. 보건의료 전공과목들 중 해부학의 기초적 배경지식은 여러 직종의 시험뿐 아니라 전공과목의 이해도 완성을 위해서도 중 요하다. 특히 임상병리학과, 물리치료학과, 작업치료학과 학생 들은 몸의 구조와 기능을 알 수 있는 해부학의 질 높은 교육은 필 수적이라 할 수 있다. 그중 해부학의 실습 중 하나인 시신을 이용 한 교육은 몸의 실제적 구조를 이해하고 자극 및 실제 병변을 확 인 가능하며 눈을 통하여 확인할 수 있으며 흥미와 재미를 느낄 수 있는 교육이다[3].

이전 보고에서 책을 통해 습득하는 이론 위주의 교육 보다 교 구재 및 사체 등을 이용한 교육의 효과가 좋다는 연구가 있으며, 사체를 이용한 해부학적 교육 후 지식 정도에 상관관계가 있다 고 보고되었고[4] 여러 대학병원의 임상교수들 또한 사체해부 의 중요성을 강조 해왔다[5]. 따라서 체계적이고 우수한 임상병 리사, 물리치료사, 작업치료사의 양성을 위해서 다양한 노력과 새로 제안된 방향 및 검토 결과를 토대로 유능한 의료 기술자를 배출하기 위한 효과적인 방법을 찾기 위한 노력도 진행 중이다[6].

보건의료계열의 해부학 수업의 질적 향상을 위해서 다양하 고 참여도 높은 교육 방법을 제시하고 연구하는 것은 교육을 담 당하는 교수자의 숙제이며 항상 고민해야 할 부분이었다[9]. 그 중 기존의 해부학 교육과 카데바실습 만족도, 이해도, 인식을 조 사하여 여러 가지 문제점을 극복해 보고자 하였으며 또한 카데 바실습 참여 후 학생들이 해부학 교육의 만족도와 인식 변화에 어떤 영향이 있는지 조사의 필요성이 있다.

본 연구자는 보건의료계열 중 임상병리과, 물리치료과, 작업 치료과 학생들을 대상으로 교과서 중심의 해부학 수업에 대한 인식조사를 통한 문제점과 해결 방안을 모색하고 기존 해부학 수업에 대한 이해도, 만족도를 조사하여 현재 학생들의 기대 수 준을 조사하며 카데바실습에 참여 후 학생들의 해부학 교육의 만족도 및 인식도, 이해도를 통하여 수업의 개선 방편으로 활용 하는 것이 연구의 목적으로 하였다. 특히 해부학적 계통별로 해 부학적 구조적 위치와 명칭을 외우는 기존의 수업 방식을 개선 뿐만 아니라 보건의료에서 전문가로 활약할 학생들에게 질 높 고 실제적 해부학 교육을 위한 연구를 진행하였다.

\section{재료 및 방법}

\section{1. 연구대상 및 자료수집 방법}

본 연구는 2017년 인체해부학을 배우는 D 대학 해부학 교육
을 받은 1 3학년 임상병리학과 20명, 물리치료학과 75명, 작 업치료학과 35 명, 총학생 130 명을 대상으로 카데바 실습 전 후 자기 보고식 설문지 조사를 시행하였다. 본 연구의 대상자 모두 에게 연구의 목적을 설명하였고, 설문지 및 평가지 결과는 연구 이외의 목적으로는 사용하지 않을 것이며 참여자는 연구에 자 유롭게 참여할 수 있어 언제라도 참여자가 원하지 않으면 설문 지 및 평가지 작성을 중단할 수 있음을 설명하였다.

\section{2. 측정도구 방법}

본 연구에서 자기보고식 설문지하였다. 자기보고식 설문지 는 Kim [10]이 사용한 골학실습의 흥미도와 이해도에 대한 설 문 문항을 변용하였으며 자기보고식 설문지에는 카데바실습 전.후 카데바실습의 필요성, 실물실습의 필요성을 함께 조사하 여 카데바실습에 대한 인식을 비교하였다. 그밖에 연구 대상자 의 일반적인 특성(학과명 및 성별), 교과서 중심의 해부학 수업 에 대한 인식조사, 해부학 수업에 대한 이해도, 만족도 등도 전 과 후를 나누어 설문을 진행하였다. 설문지는 리커트(Liker) 5 단위를 이용한 설문을 사용하였지만, 자료는 '도움이 된다, 매 우 도움이 된다, 재미있다, 매우 재미있다, 쉽다, 매우 쉽다'를 긍 정 의미, '필요치 않다, 전혀 필요치 않다, 재미없다, 매우 재미없 다, 어렵다, 매우 어렵다'를 부정 의미로 데이터를 나누어 자를 분석하였다.

\section{3. 통계분석}

분석은 윈도용 프리즘 5 (GraphPad Software, La Jolla, CA, USA)와 SPSS 18.0 (SPSS Inc., Chicago, USA)를 사용하였다. 첫째, 대응표본 t검정(paired t-test) 위해 설문을 실습전 - 후 비 교 결과와 카데바실습 후 자료 분석은 독립 표본 t검정 (independent samples t-test)하였다. 둘째, 회귀분석(regression analysis)으로 교차비(odds ratio)를 도출하였다. 모든 분석의 유의도 수준 0.05 로 설정하였다[10].

\section{결 과}

\section{1. 일반적인 특성}

연구대상자는 물리치료학과 학생 75명(57.7\%), 작업치료학 과 학생 55명(26.9\%), 임상병리학과 학생 20명(15.4\%) 총 130 명을 대상으로 하였으며, 대상자 중 남자는 31 명(23.8\%), 여자 는 99명(76.2\%)이었다. 학년별로는 1학년이 28명(21.5\%), 2학 년 55명(42.3\%), 3학년 47명(36.2\%)를 차지하였다. 그리고 고 등학교 때 생물을 이수한 학생이 79명(60.8\%), 이수하지 않은 
학생이 51명(39.2\%)이었다.

\section{2. 교과서 중심의 해부학 수업에 대한 인식조사}

자기 보고식 설문을 통해, 교과서 중심의 해부학 만족도를 살 펴보았다. 분석결과, 해부학 수업에 대한 수업의 만족도는 성별 에 따른 차이가 없었다(Table 1). 또한 전공에 따른 교과서 중심 의 해부학 수업에 대한 인식 차이가 없었으며, 모두 과에서 보통 이상의 만족도를 보였다(Table 2).

\section{3. 해부학 수업에 대한 이해도, 만족도}

교과서 중심의 해부학 이해도, 만족도를 조사하였다. 분석 결 과, 해부학 수업에 대한 수업의 만족도나 이해도는 성별에 따른 차이가 없었다 (Table 3). 그리고 전공에 따른 해부학 수업의 이 해도의 차이 역시 없었다(Table 4). 다만, 전공에 대한 만족도은 임상병리학과에서 유의하게 높았다 $(p<0.05)$.

Table 1. Satisfaction of textbook-oriented anatomy course by student sex

\begin{tabular}{|c|c|c|c|c|}
\hline \multirow{2}{*}{ Content of the questionnaire } & \multirow{2}{*}{ Classification } & \multicolumn{3}{|c|}{ Classification by student sex persons (\%) } \\
\hline & & Male & Female & Odds ratio \\
\hline \multirow[t]{3}{*}{ Satisfaction level of textbook lessons } & Positive & $18(58)$ & $47(47)$ & 1.071 \\
\hline & Intermediate & $11(35)$ & $60(50)$ & \\
\hline & Negative & $2(7)$ & $2(3)$ & \\
\hline
\end{tabular}

Table 2. Satisfaction of textbook-oriented anatomy course by department affiliation student

\begin{tabular}{|c|c|c|c|c|}
\hline \multirow{2}{*}{ Content of the questionnaire } & \multirow{2}{*}{ Classification } & \multicolumn{3}{|c|}{ Classification by department affiliation persons (\%) } \\
\hline & & PT & OT & BLS \\
\hline \multirow[t]{3}{*}{ Satisfaction level of textbook lessons } & Positive & $32(43)$ & $22(63)$ & $11(55)$ \\
\hline & Intermediate & $39(62)$ & $13(37)$ & $9(45)$ \\
\hline & Negative & $4(5)$ & $0(0)$ & $0(50)$ \\
\hline
\end{tabular}

Abbreviations: PT, Physical Therapy; OT, Occupational Therapy; BLS, Biomedical Laboratory Science.

Table 3. Satisfaction of textbook-oriented anatomy course by student sex

\begin{tabular}{|c|c|c|c|c|}
\hline \multirow{2}{*}{ Content of the questionnaire } & \multirow{2}{*}{ Classification } & \multicolumn{3}{|c|}{$\begin{array}{c}\text { Classification by student sex } \\
\text { persons }(\%)\end{array}$} \\
\hline & & Male & Female & Odds ratio \\
\hline \multirow[t]{3}{*}{ Understanding of anatomy class } & Positive & $7(23)$ & $27(27)$ & 1.031 \\
\hline & Intermediate & $22(70)$ & $55(55)$ & \\
\hline & Negative & $34(7)$ & $17(18)$ & \\
\hline \multirow{3}{*}{ Satisfaction of anatomy class } & Positive & $14(45)$ & $41(41)$ & 1.040 \\
\hline & Intermediate & $15(49)$ & 49 (49) & \\
\hline & Negative & $2(6)$ & $9(10)$ & \\
\hline
\end{tabular}

Table 4. Satisfaction of textbook-oriented anatomy course by department affiliation student

\begin{tabular}{|c|c|c|c|c|}
\hline \multirow{2}{*}{ Content of the questionnaire } & \multirow{2}{*}{ Classification } & \multicolumn{3}{|c|}{ Classification by department affiliation persons (\%) } \\
\hline & & PT & OT & BLS \\
\hline \multirow[t]{3}{*}{ Understanding of anatomy class } & Positive & $11(15)$ & $13(37)$ & $10(34)$ \\
\hline & Intermediate & $51(68)$ & $18(51)$ & $8(40)$ \\
\hline & Negative & $13(17)$ & $4(12)$ & $2(10)$ \\
\hline \multirow[t]{3}{*}{ Satisfaction of anatomy class } & Positive & $22(29)$ & $16(46)$ & $17(82)$ \\
\hline & Intermediate & $45(60)$ & $16(46)$ & $3(15)$ \\
\hline & Negative & $8(11)$ & $3(8)$ & $0(0)$ \\
\hline
\end{tabular}

Abbreviations: 'See table 2.' 
492 Se-Hoon Jung and Seung-Ju Ahn. Perception and Satisfaction Survey of Human Anatomy Dissection Course Taught by Health Science

Table 5. Satisfaction of cadaver dissection by student sex

\begin{tabular}{|c|c|c|c|c|}
\hline \multirow{2}{*}{ Content of the questionnaire } & \multirow{2}{*}{ Classification } & \multicolumn{3}{|c|}{ Classification by student sex persons (\%) } \\
\hline & & Male & Female & Odds ratio \\
\hline \multirow[t]{3}{*}{ Interest in cadaver dissection } & Positive & $28(29)$ & $93(93)$ & \\
\hline & Intermediate & $3(10)$ & $6(7)$ & 0.883 \\
\hline & Negative & $2(7)$ & $2(3)$ & \\
\hline \multirow[t]{3}{*}{ Understanding cadaver dissection } & Positive & $28(90)$ & $94(94)$ & \\
\hline & Intermediate & $3(10)$ & $5(6)$ & 0.751 \\
\hline & Negative & $2(6)$ & $9(10)$ & \\
\hline \multirow[t]{3}{*}{ Degree of help of class } & Positive & $31(8)$ & $98(99)$ & \\
\hline & Intermediate & $0(0)$ & $1(1)$ & * \\
\hline & Negative & $0(0)$ & $0(0)$ & \\
\hline
\end{tabular}

*The odds ratio can not be obtained with a significance probability of 1 .

Table 6. Satisfaction of cadaver dissection by department affiliation student

\begin{tabular}{|c|c|c|c|c|}
\hline \multirow{2}{*}{ Content of the questionnaire } & \multirow{2}{*}{ Classification } & \multicolumn{3}{|c|}{ Classification by department affiliation persons (\%) } \\
\hline & & PT & OT & BLS \\
\hline \multirow[t]{3}{*}{ Interest in cadaver dissection } & Positive & $75(100)$ & $34(97)$ & $20(100)$ \\
\hline & Intermediate & $0(0)$ & $1(3)$ & $0(0)$ \\
\hline & Negative & $0(0)$ & $0(0)$ & $0(0)$ \\
\hline \multirow{3}{*}{ Understanding cadaver dissection } & Positive & $70(93)$ & $33(94)$ & $19(95)$ \\
\hline & Intermediate & $5(7)$ & $2(6)$ & $1(5)$ \\
\hline & Negative & $0(0)$ & $0(0)$ & $0(0)$ \\
\hline \multirow[t]{3}{*}{ Degree of help of class } & Positive & $70(93)$ & $32(91)$ & 19 (95) \\
\hline & Intermediate & $5(7)$ & $3(9)$ & $1(5)$ \\
\hline & Negative & $0(0)$ & $0(0)$ & $0(0)$ \\
\hline
\end{tabular}

Abbreviations: 'See table 2.'

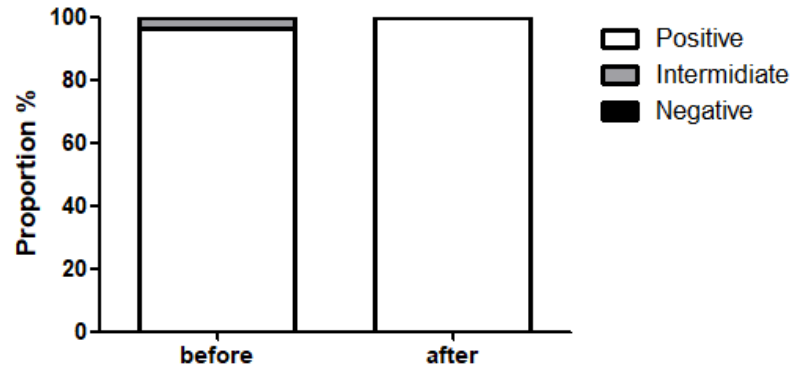

Figure 1. Survey on the necessity of cadaver dissection.

\section{4. 카데바실습에 대한 인식조사}

카데바실습 전 ·후의 카데바실습에 대한 인식조사를 하였 다. 분석결과 대부분의 학생들은 성별이나 고등교육 교과과정 과 관계없이 카데바실습이 해부학수업에 꼭 필요하다고 인식하 였고(Table 5), 실습 후 카데바실습이 꼭 필요하다고 생각했다 (Figure 1). 또한 수업의 도움 정도에서도 높은 비율로 도움이 될 것으로 조사되었다(Table 6).

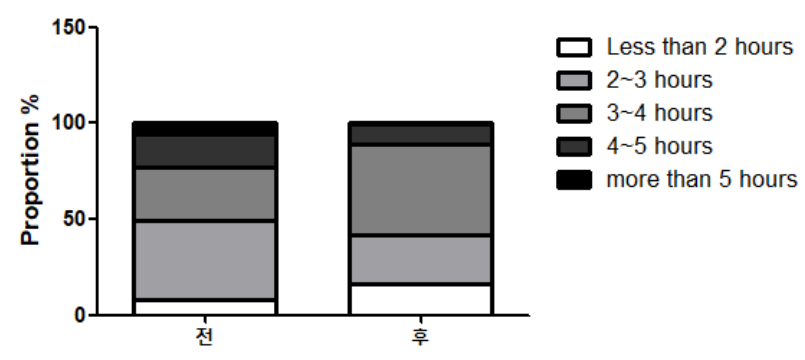

Figure 2. Ideal practice time for cadaver dissection.

\section{5. 실물실습에 대한 인식조사}

본 연구에서는 카데바실습 후에도 실물실습이 필요한지 조 사할 목적으로 실물실습 희망여부, 희망 시 실습시간 및 비용을 설문하였다. 조사결과 모든 학생이 카데바실습 후에도 실물실 습이 필요하다고 인식하였고 3 4시간이 가장 적당하다고 생 각했다(Figure 2). 


\section{고 찰}

진화되고 있는 의학의 발달로 치료와 의료 서비스에 대한 기 대가 증가되고 있으며 의료종사자들에게 보다 더 체계적이고 전문적인 지식과 기술을 요구하고 있는 실정이다[7]. 이러한 의 료의 발전의 바탕에는 기초의학 발전이 중요하며 기초과목들 중에서 해부학은 인체의 구조와 형태를 알 수 있는 의학 과목의 핵심이라 할 수 있다. 보건의학계열을 중심으로 해부학 교육의 질적 향상을 위해서 여러 가지 교육 방법을 개발하기 위한 노력 이 진행되고 있는데 특히 실습교육은 교육 효과 전달이 우수하 여 중요성이 강조되는 바, 그중 카데바 실습(cadaver dissection)이 우수하다고 알려져 있다[8].

그러나 보건의료계열 종사자로서 해부학 과목의 중요성은 매우 높음에도 불구하고 피교육자 입장에서는 전문용어와 방대 한 양의 암기로 인해 교육 전달 효과 매우 떨어지는 실정이다. 이 에 본 연구는 임상병리학과, 물리치료학과, 작업치료학과 학생 을 대상으로 카데바실습이 해부학교수 과정에서의 중요성과 필 요성 등을 알아보고자 하였다. 대상 학생들은 기존의 교과서를 통한 해부학을 이수한 학생들 중 카데바실습을 희망한 학생들 로 교육전과 후의 만족도 및 인식도를 조사하였다. 그 결과 성별 및 전공에 따른 해부학 수업의 인식 차는 없는 것은(Table 5, 6) 설문지의 다양성 부족 및 각 학과별 맞춤 설문내용의 부족이 문 제라 생각된다. 다만 임상병리학과에서 유의하게 높았는데, 수 업의 보통 이상이라는 만족도, 이해도는 아마 한정된 수업으로 비교 대상이 없었던 학생들의 한계점을 반영하지 못한 결과라 할 수 있다(Table 3, 4). 또한 물리치료학과, 작업치료학과 학생 들의 해부학 수업은 임상병리학과에 비하여 다양한 커리큘럼으 로 구성되어 있고 해부학의 중에서도 근골격계의 심층 수업이 이런 결과를 가져온 것으로 생각된다. 그리고 수강신청 시 해부 학 교육의 기대치와수업에 대한 만족도에 비해 수강 종료 시 만 족도가 현저히 떨어진다는 응답이 많았는데 이는 해부학 수업 의 내용이 이론 중심으로 되어 있고 복잡한 인체의 구조와 명칭 을 의학용어로 혹은 영문으로 배우고 있으며 많은 부분을 암기 해야 하는 과목으로 학생들의 수업의 인식도에 비해 만족도가 못 미치고 있는 것이 대부분의 현실이 반영된 것으로 생각된다.

최근 연구에서 보건의학계열 임상병리학과 학생을 대상으로 해부학 수업의 개선을 위해서 골학 실습으로 뼈의 구조와 기능 을 이해하는 학습 후 골학에 대한 학습 효과가 향상된 연구에서 처럼 이론으로 교육하는 것보다 실습을 통한 수업은 검증된 바 있다[10]. 또한 해부학 교육은 초기 학습, 망각, 재건축, 적용의 4단계가 있으며 그중 초기 단계의 학습에 카데바실습 및 해부학
교구 구조물을 이용한 수업 방식이 유용하다고 보고된 바 있다 [11]. 본 연구에서는 대부분의 학생들이 실물실습 시간 3-4시간 이 더 필요하다고 조산된 결과(Figure 2)는 해부학적 지식 습득 방법으로 카데바실습 및 실물을 이용한 수업이 학습효과의 증 대와 추후 임상적 활용에서 가치가 높기에 나타난 결과로 사료 된다. 임상병리학과, 물리치료학과, 작업치료학과 학생들의 해 부학 교육은 1학년 학기 초에 교과과정이 되어 있고 학습에 대 한 호기심과 열정이 높다[12]. 그래서 학생들의 학습 효과를 위 해서도 기존의 수업 방식을 유지하기보다 새로운 수업 방식을 소개의 방편으로도 다음과 같이 카데바실습 참여수업 또는 내 용에 맞는 다양한 실물 교구들을 이용한 수업방식의 노력들이 해부학 수업의 질적 향상 가져올 수 있으며 어렵게만 느끼던 수 업시간이 흥미롭고 즐거운 시간이 될 수 있을 것이라 생각한다. 그러나 임상병리학, 물리치료학과, 작업치료학과 학생들이 카 데바실습 및 시신의 보존에 관한 법률적 제한과 카데바실습을 전문적으로 진행할 교수진 및 실습실 운영할 상황이 되지 못하 는 실정이다[13]. 그럼에도 불구하고 해부학의 교육의 질적 향 상을 위해서라도 새로운 교육 방법 찾는 노력은 계속되어야 한 다[14]. 그런 면에서 전문 카데바실습을 운영하는 의과대학 및 전문기관의 협력을 통하거나 여건이 된다면 해외의 사체 실습 프로그램에 참여하는 방법이 좋은 대안이 되리라 생각되며 연 구결과에서처럼 학생들이 실물 해부의 요구도 또한 높았었다. 이러한 문제점의 해결을 위해서라도 다양한 조사를 통하여 학 생들이 원하는 교육이 무엇인지 항상 고민되어야 하며 활용가 치가 있는 교육을 개발을 해야 한다[15].

본 연구자는 여러 방면으로 진행되고 있는 해부학 교육의 개 선을 위한 연구들처럼 실질적인 교육의 효과를 위해서 카데바 실습 및 실물 실습을 제안해 본다. 하지만 여러 어려움들이 있으 며 시행의 여러 문제점들이 있다. 그럼에도 불구하고 지역의 보 건의료 교육의 실질적 학습 및 교육을 위해 보건의료계열 학생 들이 지역 해부 교육센터, 각 지역 의과대학의 협력을 통한 교육 인프라를 구축하는 것이 지역 보건의료학과의 교육의 질을 개 선할 수 있는 방안이 될 것이라고 생각된다. 이번 연구의 결과로 모든 학생들의 해부학 교육의 제언을 위한 일반화는 될 수 없지 만 보건계열 학과 중 임상병리학과, 물리치료학과, 작업치료학 과 학생들의 대표성이 있다고 생각되며 앞으로 다양한 연구가 진행되어야 할 필요성이 있다. 해부학 교육은 보건의료계열 학 생들이 전공과목을 습득하는데 있어 가장 중추적 역할을 하는 과목이다. 그러므로 이번 연구에서 조사된 결과를 바탕으로 다 양하며 학생들의 흥미를 유도하는 만족도 높은 교육이 되기 위 한 기초자료로 활용되기를 기대하는 바이다. 


\section{요 약}

해부학 교육은 보건의학계열 학생들에게 가장 기본이 되는 과목이며 중요하다. 해부학 교육의 질적 개선 및 다양성을 위해 서 임상병리학과, 물리치료학과, 작업치료학과 학생 130 명을 대상으로 설문을 통해 기존의 해부학 교육과 카데바실습 (cadaver dissection)의 만족도, 이해도, 인식을 조사하였다. 분 석 결과, 해부학 수업에 대한 수업의 만족도는 성별에 따른 차이 가 없었다. 또한 전공에 따른 교과서 중심의 해부학 수업에 대한 인식 차이가 없었으며, 모두 과에서 보통 이상의 만족도를 보였 다. 그리고 전공에 따른 해부학 수업의 이해도의 차이 역시 없었 다. 다만, 전공에 대한 만족도은 임상병리학과에서 유의하게 높 았다 $(p<0.05)$. 카데바실습 전. 후의 카데바실습에 대한 인식 분석결과 대부분의 학생들은 성별이나 고등교육 교과과정과 관 계없이 카데 실습이 해부학수업에 꼭 필요하다고 인식하였고, 실습 후 카데바실습이 꼭 필요하다고 생각했으며 조사결과 모 든 학생이 카데 실습 후에도 실물실습이 필요하다고 인식하였 고 3 4시간이 가장 적당하다고 생각했다. 이번 결과로 해부학 교육의 다양성을 위한 학생들의 요구도가 높았으며 카테바실습 및 실물을 이용한 실습을 이용한 해부학 교육이 학습 효과가 높 을 것으로 기대 된다.

\section{Acknowledgements: None}

\section{Funding: None}

Conflict of interest: None

\section{REFERENCES}

1. Coulehan JL, Williams PC, Landis D, Naser C. The first patient: reflections and stories about the anatomy cadaver. Teaching and Learning in Medicine. 1995;7(1):61-66.

2. Son IH, Jeon MJ, Jung GB. The effect of education in anatomy using cadavers to the paramedic students. The Journal of the
Korea Contents Association. 2013;3(2):341-342.

3. Older J. Anatomy: a must for teaching the next generation. Surgeon. 2004;2:79-90.

4. Craig S, Tait N, Boers D, McAndrew D. Review of anatomy education in Australian and New Zealand medical schools. ANZ Journal of Surgery. 2010;80(4):212-216.

5. Im SJ, Gam BS, Lee SY, Woo JS, Lee JT, Lee SH, Im H, Jo KJ, Baek SY. "Study of clinical medical teachers" attitudes to the knowledge of gross anatomy of medical students. Korean J Phys Anthropol. 2014;27(4):211-218.

6. Kim HS, Kwon PS, Kang JH, Yang MG, Park JO, Kim DJ, et al. Survey on the education system and national licensing examination for fostering competent medical technologists. Korean J Clin Lab Sci. 2017;49(2):161-170.

7. Yoo HH, Song CH, Han EH, Kim HT. The effect of education program of cadaver dissection for the paramedical students. Korean J Phys Anthropol. 2014;27(3):145-154.

8. Kim DH. The effect and suggestions for operation of cadaver dissection program for dental hygiene students. Korea J Phys Anthropol. 2015;28(4):213-221.

9. Park JY, Park SM. Influence of college adjustment and social support on career development level of college students who majors in health or social welfare. Korean J Clin Lab Sci. 2017;49:180-186.

10. Kim JH. Case study on osteology practice at human anatomy course: focused on the students in the department of biomedical laboratory science. The Journal of the Korea Contents Association. 2016;16(4):144-150.

11. Smith CF, Mathias HS. What impact does anatomy education have on clinical practice? Clin Anat. 2011;24(1):113-119.

12. Song CW, Lee BY, Koh KS. The application of the dissection seminar in anatomical practice. Korean J Phys Anthropol. 2009; 22(4):287-294.

13. Kim DI. Questionnaire survey analysis on necessity of cadaveric dissection for nursing students: in scope of nursing students and professors. Korean J Phys Anthropol. 2015;28(2):119-125.

14. Lee CH, Kim DJ, Choi JS, Lee JW, Lee MW, Jo JW, et al. An estimation of the efficiency and satisfaction for EEG practice using the training 10-20 electrode system: A questionnaire survey. Korean J Clin Lab Sci. 2017;49(3):300-307.

15. Jung SH. A study on educational requirement of forensic medicine for biomedical Laboratory Science. Korean J Clin Lab Sci. 2015;47(3):153-158. 